\title{
Coherent $v-N$ scattering and the search for physics beyond the standard model
}

\author{
J. Barranco \\ Instituto de Física, Universidad Nacional Autónoma de México, Apdo. Postal 20-364, 01000 \\ México D.F., México.
}

\begin{abstract}
We focus in future proposals to measure coherent neutrino-nuclei scattering and we show that such kind of experiments are very sensitive to nonstandard neutrino interactions with quarks. First in a model independent parametrization and then we focused in particular models such as leptoquarks and models with extra neutral gauge bosons and with R-parity breaking interactions. We show that in all these three different types of new physics it is possible to obtain competitive bounds to those of future collider experiments. For the particular case of leptoquarks we found that the expected sensitivity to the coupling and mass for most of the future experimental setups is quite better than the current constraints.
\end{abstract}

Keywords: Nonstandard neutrino interaction

\section{THE COHERENT NEUTRINO-NUCLEUS CROSS SECTION}

There are few predictions made by the Standard Model (SM) that have not yet been observed, most of the times due to experimental difficulties. One of these non-observed predictions is the so called coherent neutrino-nucleus scattering [1]. In this process a neutrino scatters coherently not only the nucleons but the nucleus itself. The coherent scattering requires momentum transfer, $q$, small compared with the inverse nucleus size, $q \leq 1 / R, R$ the nuclear radius. This condition is well satisfied for almost every typical atom, in the case of neutrino energies coming from reactors, artificial sources, the sun or supernovas. Neglecting radiative corrections the cross section is

$$
\frac{d \sigma}{d T}=\frac{G_{F}^{2} M}{2 \pi}\left\{\left(G_{V}+G_{A}\right)^{2}+\left(G_{V}-G_{A}\right)^{2}\left(1-\frac{T}{E_{V}}\right)^{2}-\left(G_{V}^{2}-G_{A}^{2}\right) \frac{M T}{E_{V}^{2}}\right\},
$$

where $M$ is the mass of the nucleus, $T$ is the recoil nucleus energy, $E_{v}$ is the incident neutrino energy and the axial and vector couplings are

$$
\begin{aligned}
G_{V} & =\left[g_{V}^{p} Z+g_{V}^{n} N\right] F_{n u c l}^{V}\left(q^{2}\right), \\
G_{A} & =\left[g_{A}^{p}\left(Z_{+}-Z_{-}\right)+g_{A}^{n}\left(N_{+}-N_{-}\right)\right] F_{n u c l}^{A}\left(q^{2}\right) .
\end{aligned}
$$

$Z$ and $N$ represent the number of protons and neutrons in the nucleus, while $Z_{ \pm}\left(N_{ \pm}\right)$ stands for the number of protons (neutrons) with spin up and spin down respectively. The vector and axial nuclear form factors, $F_{n u c l}^{V}\left(q^{2}\right)$ and $F_{n u c l}^{A}\left(q^{2}\right)$, are usually assumed to be equal and of order of unity in the limit of small energies, $q^{2} \ll M^{2}$. The SM neutral 


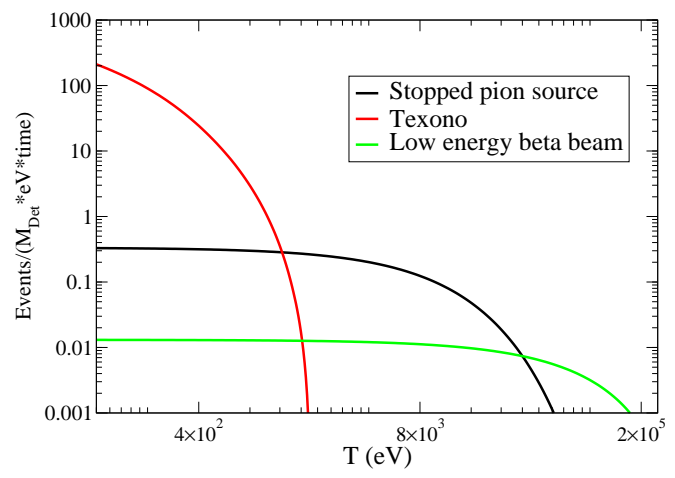

FIGURE 1. Events vs. energy for three different experimental proposals

current vector couplings of neutrinos with protons, $g_{V}^{p}$, and with neutrons, $g_{V}^{n}$, are

$$
\begin{aligned}
& g_{V}^{p}=\rho_{v N}^{N C}\left(\frac{1}{2}-2 \hat{\kappa}_{v N} \hat{s}_{Z}^{2}\right)+2 \lambda^{u L}+2 \lambda^{u R}+\lambda^{d L}+\lambda^{d R}, \\
& g_{V}^{n}=-\frac{1}{2} \rho_{v N}^{N C}+\lambda^{u L}+\lambda^{u R}+2 \lambda^{d L}+2 \lambda^{d R} .
\end{aligned}
$$

Here $\hat{s}_{Z}^{2}=\sin ^{2} \theta_{W}=0.23120, \rho_{v N}^{N C}=1.0086, \hat{\kappa}_{v N}=0.9978, \lambda^{u L}=-0.0031, \lambda^{d L}=$ -0.0025 and $\lambda^{d R}=2 \lambda^{u R}=7.5 \times 10^{-5}$ are the radiative corrections given by the PDG [2]. The axial contribution can be neglected as can be seen from eq. (1) since the ratio of axial to vector contribution is expected to be of the order $1 / A, A$ the atomic number. The spin-zero cross section of electron neutrino scattering off nucleus in the low energy limit, $T \ll E_{V}$ is

$$
\frac{d \sigma}{d T}\left(E_{V}, T\right)=\frac{G_{F}^{2} M}{\pi}\left(1-\frac{M T}{2 E_{V}^{2}}\right)\left[Z\left(g_{V}^{p}\right)+N\left(g_{V}^{n}\right)\right]^{2} .
$$

The number of expected events, neglecting for a moment the detector efficiency and resolution, can be estimated as

$$
N_{\text {events }}=t \phi_{0} \frac{M_{\text {detector }}}{M} \int_{E_{\min }}^{E_{\max }} d E_{v} \int_{T_{t h}}^{T_{\max }\left(E_{v}\right)} d T \lambda\left(E_{v}\right) \frac{d \sigma}{d T}\left(E_{v}, T\right)
$$

with $t$ the data taking time period, $\phi_{0}$ the total neutrino flux, $M_{\text {detector }}$ the total mass of the detector, $\lambda\left(E_{v}\right)$ the normalized neutrino spectrum, $E_{\max }$ the maximum neutrino energy and $T_{t h}$ the detector energy threshold. One can see that the maximum nuclei's recoil energy depends on nucleus mass $M$ through the relation $T_{t h}^{\max }=2 E_{v}^{\max ^{2}} /\left(M+2 E_{v}^{\max }\right)$. This is the biggest experimental challenge to defeat in order to detect this process. For example, reactor neutrino with $E_{v}^{\max }=10 \mathrm{MeV}$ produces a maximum nucleus recoil 
TABLE 1. Expected events for different experimental setups

\begin{tabular}{cccc}
\hline Experiment & $M_{0}$ & Expected events/yr & systematic error estimate \\
\hline Texono, $E_{t h}=400 \mathrm{eV}$ & $1 \mathrm{~kg}, \mathrm{Ge}$ & 3790 & $2 \%$ \\
Texono, $E_{t h}=100 \mathrm{eV}$ & $1 \mathrm{~kg}, \mathrm{Ge}$ & 25196 & $2 \%$ \\
Beta beam, $E_{t h}=15 \mathrm{keV}$ & $1 \mathrm{ton}, \mathrm{Xe}$ & 1390 & $2 \%$ \\
Beta beam, $E_{t h}=5 \mathrm{keV}$ & $1 \mathrm{ton}, \mathrm{Xe}$ & 5309 & $2 \%$ \\
Stopped pion, $E_{t h}=10 \mathrm{keV}$ & $100 \mathrm{~kg}, \mathrm{Ne}$ & 627 & $5 \%$ \\
\hline
\end{tabular}

for Germanium of $12 \mathrm{KeV}$. Nevertheless, experiments that look for direct dark matter searches have improved the detectors at very low threshold. Different proposals have been suggested to detect the coherent $v-N$ scattering that differs each other depending on the neutrino source and the detector properties.

The experimental proposals:

TEXONO: Texono collaboration has recently started a research program towards the measurement of neutrino-nuclei coherent scattering by using reactor neutrinos and $1 \mathrm{Kg}$ of an "ultra-low-energy" germanium detector (ULEGe) [3] with a threshold as low as $100 \mathrm{eV}$ and we have consider also a threshold of $400 \mathrm{eV}$. We can consider that the error will be dominated by the reactor power, its fuel composition, and the anti-neutrino spectrum. We assume that these uncertainties will give an approximate error of $2 \%$ [4] and one year of data taking.

Stopped pion source: Another proposal for detecting the coherent neutrino-nucleus scattering considers the use of another source of neutrinos, a stopped pion source (SPS), such as the Spallation Neutron Source at Oak Ridge National Laboratory [5]. The neutrino spectra are well known. Here we will consider only the total delayed flux $\left(v_{e}+\overline{v_{\mu}}\right)$ that comes from pion decay. We assume a total flux of $\sim 10^{7} v \mathrm{~s}^{-1} \mathrm{~cm}^{-2}$. Among different possible detector materials such as Ar, Ge or Xe, we will concentrate on the noble gas detector, ${ }^{20} \mathrm{Ne}$, of typical mass about $100 \mathrm{~kg}$ with a data taking time scale from one to several years and a threshold of $10 \mathrm{keV}$.

Low energy Beta-Beams: The usage of accelerated radioactive nuclei to produce a well known flux of neutrinos - beta beam - was proposed in [6]. It was shown soon afterwards that low energy beta beams open new possibilities to study neutrino properties [7] and, recently, a neutrino-nuclei coherent scattering experiment using neutrinos from low energy beta beams was discussed [9]. In particular we base our analysis on the betabeam experiment discussed in $[8,9]$. The number of expected neutrinos observed in this proposal is

$$
N_{\text {events }}^{\beta-\text { beam }}=\operatorname{tg} \tau n h \times \int_{0}^{\infty} d E_{v} \Phi_{\text {tot }}\left(E_{v}\right) \sigma\left(E_{v}\right),
$$

where $t=1$ year is the data taking time, $n$ is the number of target nuclei per unit volume, $\sigma\left(E_{v}\right)$ is the relevant neutrino-nucleus cross-section. We considered the case of a ton of $\mathrm{Xe}$ as a target and a factor $\gamma=14$ for ${ }^{6} \mathrm{He}$ ions as described in Ref. [9]. As for the threshold energy, we consider both the realistic threshold of $15 \mathrm{keV}$ where background events are negligible as well as the very optimistic $5 \mathrm{keV}$ threshold that, according to the same reference, will give a bigger number of events if background could be subtracted, though at present there is no technology capable of dealing with such a background. The total neutrino flux through detector is given in [8]. 
TABLE 2. Constrains in NSI parameters with Texono-like experiment at 90\% C.L. Present limits and $v$ Factory sensitivity are taken from [10]. For details see [14]

\begin{tabular}{cccc}
\hline Present Limits & ${ }^{76} \mathrm{Ge} T_{T h}=400 \mathrm{eV}$ & ${ }^{76} \mathrm{Ge} T_{T h}=100 \mathrm{eV}$ & $v$ Factory \\
\hline$-0.5<\varepsilon_{e e}^{d V}<1.2$ & $\left|\varepsilon_{e e}^{d V}\right|<0.003$ & $\left|\varepsilon_{e e}^{d V}\right|<0.001$ & $\left|\varepsilon_{e e}^{d V}\right|<0.001$ \\
\hline$-1.0<\varepsilon_{e e}^{u V}<0.61$ & $\left|\varepsilon_{e e}^{u V}\right|<0.002$ & $\left|\varepsilon_{e e}^{u V}\right|<0.001$ & $\left|\varepsilon_{e e}^{u V}\right|<0.002$ \\
\hline
\end{tabular}

\section{THE NON STANDARD INTERACTION}

We can go a step further and look for new physics in the neutrino sector. New Physics such as non standard interactions (NSI) that can be included adding to the Standard Model (SM) Lagrangian the effective Lagrangian [10]

$$
-\mathscr{L}_{\mathrm{NSI}}^{e f f}=\varepsilon_{\alpha \beta}^{f P} 2 \sqrt{2} G_{F}\left(\bar{v}_{\alpha} \gamma_{\rho} L v_{\beta}\right)\left(\bar{f} \gamma^{\rho} P f\right)
$$

where $f$ is a first generation SM fermion: $e, u$ or $d$, and $P=L$ or $R$. That kind of NSI Lagrangian appear in a natural way for example when it is added mass to the neutrinos [11] and also appear from super-symmetric theories [12]. So, all new physics will be included in the effective parameter $\varepsilon_{\alpha \beta}^{f P}$ and the goal is to constrain such parameters using the neutrino data. But the solar and atmospheric data allow big values on such NSI parameters and even worst, the appearance of new "dark" solutions [13]. It turns now relevant to really understand correctly the NSI parameters if a good determination in the mixing parameters is wanted. Experiments at low energy offer an excellent opportunity for the NSI study, because the no dependence in neutrino mixing parameters. In particular, the coherent neutrino-nucleus scattering can set strong bounds in NSI parameters because the enhancement in the number of detectable events as can be seen in Table 1.

The NSI in $v-N$ scattering: The coherent neutrino-nucleus scattering cross section after the addition of Lagrangian 8 is easily obtained with the change in the coupling constant $g_{v}^{p} \rightarrow g_{v}^{p}+2 \varepsilon_{V}^{u}+\varepsilon_{V}^{d}$ and $g_{v}^{n} \rightarrow g_{v}^{n}+\varepsilon_{V}^{u}+2 \varepsilon_{V}^{d}$ in eq. (5). The expected number of events depends now in the NSI parameters $N_{\text {Events }}\left(\varepsilon_{V}^{d}, \varepsilon_{V}^{u}\right)$ and it is possible to constraint the NSI parameters by doing a $\chi^{2}$ analysis taking the SM as central value. Taking only one parameter at a time and just as illustrative we show the possible constraint obtained with a Texono-like experiment with two different threshold energies in Table 2. The sensitivity for this kind of experiments is very competitive with future $v$-factories.

Once we constrained the NSI parameters, it is natural to ask if the bounds in the effective parameter can be translated into constrains in specific models. Here we did in three different realizations of theories beyond the standard model:Heavy neutral vector bosons $Z^{\prime}$, Leptoquarks and supersymmetric theories with $R$-parity breaking terms. 
TABLE 3. Effective 4-fermion NSI parameter expressed in terms of the relevant parameters for three different models beyond the SM.

\begin{tabular}{cccc}
\hline & Heavy neutral gauge boson $Z^{\prime}$ & Leptoquarks & R-parity breaking terms \\
\hline$\varepsilon^{u V}$ & 0 & $\frac{\lambda_{u}^{2}}{m_{l q}^{2}} \frac{\sqrt{2}}{4 G_{F}}$ & 0 \\
\hline$\varepsilon^{d V}$ & $-4 \gamma \sin ^{2} \theta_{W} \rho_{v N}^{N C}\left(\frac{3 c_{\beta}}{2 \sqrt{24}}+\frac{s_{\beta}}{6} \sqrt{\frac{5}{8}}\right) \frac{c_{\beta}}{\sqrt{6}}$ & $\frac{\lambda_{d}^{2}}{m_{l q}^{2}} \frac{\sqrt{2}}{4 G_{F}}$ & $\left(\frac{M_{W}^{2}}{g^{2}}\right)\left(\frac{\left|\lambda_{1 j 1}^{\prime}\right|^{2}}{m_{\tilde{d}_{j L}}^{2}}-\frac{\left|\lambda_{1 k}^{\prime}\right|^{2}}{m_{\tilde{d}_{k R}}^{2}}\right)$ \\
\hline
\end{tabular}

\section{NSI IN DIFFERENT EXTENSIONS OF SM}

Heavy neutral vector bosons: Extra $Z^{\prime}$ bosons are predicted in string inspired extensions of the SM, in left-right symmetric models, in models with dynamical symmetry breaking, in "little Higgs" models and in certain classes of theories with extra dimensions. In many of these models it is expected that $Z^{\prime}$ mass can be around $\mathrm{TeV}$ scale. The present experimental lower limits to the neutral gauge boson mass come from the Tevatron and LEP experiments [2]. Forthcoming measurements at LHC will provide sensitivity to the $Z^{\prime}$ mass up to $5 \mathrm{TeV}[15,16]$. The effect of this $Z^{\prime}$ boson will generate a neutral current that modifies the SM coupling constants with a NSI parameters as expressed in Table 3, where $c_{\beta}=\cos \beta, s_{\beta}=\sin \beta$ and $\gamma=\left(M_{Z} / M_{Z^{\prime}}\right)^{2}$ are the relevant parameters.

Leptoquarks: A leptoquark is a scalar or vector boson that couples to a lepton and a quark. There are no such interactions in the SM, but they are expected to exist in various extensions of the SM [2], such as the Pati-Salam model [17], grand unification theories based on $S U(5)[18,19]$ and $S O(10)$ [20] gauge groups and extended technicolor models [21]. The leptoquark contribution effectively (in 4-fermion approximation) can be written as expressed in Table 3 [22] where $\lambda_{u}, \lambda_{d}$ are couplings, $m_{l q}$ is leptoquark mass. This parametrization is given for vector leptoquarks. In the case of scalar leptoquarks, our results should be multiplied by a factor $1 / 2$ [22].

$\mathbf{R}$ parity breaking terms: In supersymmetric theories, gauge invariance does not imply baryon number (B) and lepton number (L) conservation and, in general, the so called R-parity (defined as $R=(-1)^{3 B+L+2 S}$ where $S$ is the spin) is violated. However, one has to keep the consistency with the non-observation of fast proton decay. One may consider, for instance, the R-parity violating MSSM (imposing baryon number conservation) with a superpotential that contains the $L$ - violating terms [12] $\lambda_{i j k} L_{L}^{i} L_{L}^{j} \bar{E}_{R}^{k}$ ,$\lambda_{i j k}^{\prime} L_{L}^{i} Q_{L}^{j} \bar{D}_{R}^{k}$, where we use the standard notation, $L_{L}, Q_{L}, \bar{E}_{R}$, and $\bar{D}_{R}$ to denote the chiral superfields containing the left-handed lepton and quark doublets and the right-handed charged-lepton and $d$-quark singlets respectively; $i, j, k$ are generation indices. At low energies, the heavy Supersymmetry particles can be integrated out and the net effect of the $R$-breaking interactions is to generate effective 4-fermion operators involving the lepton and quark fields. By considering the case where a single Yukawa coupling (with one flavor structure) is much larger than the others, the effective four-fermion operator generated by $L_{L}^{i} Q_{L}^{j} \bar{D}_{R}^{k}$ takes the same form as in Eq. (8) with the couplings [12, 23] as expressed in Table 3. 
TABLE 4. Constraint in the relevant parameters for each model

\begin{tabular}{|c|c|c|c|c|c|c|}
\hline & Texono $_{100 e \mathrm{~V}}$ & Texono $_{400 \mathrm{eV}}$ & SPS & $\beta$-Beam $15 \mathrm{KeV}$ & $\beta-\mathrm{Beam}_{5 \mathrm{KeV}}$ & Current \\
\hline$Z^{\prime}$ & 792 & 722 & 479 & 619 & 725 & $680(\mathrm{GeV})$ \\
\hline Leptoquark & 894 & 805 & 546 & 684 & 805 & $298(\mathrm{GeV})$ \\
\hline$R$-Parity & 0.0020 & 0.0025 & 0.005 & 0.003 & 0.0024 & 0.012 \\
\hline
\end{tabular}

\section{RESULTS}

Our results are summarized in Table 4. For definiteness we have fixed some parameters. The estimated sensitivity has been calculated for the detector's mass given in the proposal and the time $=1$ year of data taking. In case of $Z^{\prime}$ we have estimated the sensitivity of each experimental proposal for the mass for the extra gauge boson in the particular case where $\cos \beta=1$, that is called the $\chi$-model. For leptoquarks we have fixed the coupling $\lambda_{q}$ to the electroweak scale $\left(\lambda_{q}^{2}=4 \pi / 137\right)$ and the estimation are on the leptoquark mass at $90 \%$ C.L. in GeV. Finally, for SUSY with $R$-parity breaking terms, the estimation is done over the difference in the coupling constants $\left|\lambda_{1 j 1}^{\prime}\right|^{2} / m_{\tilde{d}_{j L}}^{2}-\left|\lambda_{11 k}^{\prime}\right|^{2} / m_{\tilde{d}_{k R}}^{2}$ with s-quark masses normalized to $100 \mathrm{GeV}$. We can see that in most of the cases we have competitive or as in the leptoquark case much better sensitivity to the relevant parameters than the current constraints. For a more detailed discussion see [24].

Acknowledges This work was partially supported by DGAPA-UNAM.

\section{REFERENCES}

1. D. Z. Freedman, Phys. Rev. D 9, 1389 (1974).

2. W. M. Yao et al. [Particle Data Group], J. Phys. G 33 (2006) 1.

3. H. T. Wong, H. B. Li, J. Li, Q. Yue and Z. Y. Zhou, J. Phys. Conf. Ser. 39 (2006) 266

4. P. Huber and T. Schwetz, Phys. Rev. D70, 053011 (2004)

5. K. Scholberg, Phys. Rev. D 73 (2006) 033005 [arXiv:hep-ex/0511042].

6. P. Zucchelli, Phys. Lett. B 532 (2002) 166.

7. C. Volpe, J. Phys. G 30 (2004) L1

8. J. Serreau and C. Volpe, Phys. Rev. C 70 (2004) 055502

9. A. Bueno, M. C. Carmona, J. Lozano and S. Navas, Phys. Rev. D 74 (2006) 033010.

10. S. Davidson, C. Pena-Garay, N. Rius and A. Santamaria, JHEP 0303, (2003) 011

11. J. Schechter and J. W. F. Valle, Phys. Rev. D 22 (1980) 2227.

12. V. D. Barger, G. F. Giudice and T. Han, Phys. Rev. D 40 (1989) 2987.

13. O. G. Miranda, M. A. Tortola and J. W. F. Valle , arXiv:hep-ph/0406280.

14. J. Barranco, O. G. Miranda and T. I. Rashba, JHEP 0512 (2005) 021

15. M. Dittmar, A. S. Nicollerat and A. Djouadi, Phys. Lett. B 583 (2004) 111

16. T. G. Rizzo,

17. J. C. Pati and A. Salam, Phys. Rev. D 10, 275 (1974).

18. H. Georgi and S. L. Glashow, Phys. Rev. Lett. 32, 438 (1974).

19. I. Dorsner, P. F. Perez and R. Gonzalez Felipe, Nucl. Phys. B 747, 312 (2006) I. Dorsner and P. F. Perez, Nucl. Phys. B 723, 53 (2005)

20. H. Fritzsch and P. Minkowski, Annals Phys. 93, 193 (1975).

21. E. Farhi and L. Susskind, Phys. Rept. 74, 277 (1981).

22. S. Davidson, D. C. Bailey and B. A. Campbell, Z. Phys. C 61, 613 (1994)

23. M. Chemtob, Prog. Part. Nucl. Phys. 54, 71 (2005)

24. J. Barranco, O. G. Miranda and T. I. Rashba, Phys. Rev. D 76, 073008 (2007) 